\title{
Estarão alguns grupos populacionais brasileiros sujeitos à ação de disruptores endócrinos?
}

\author{
Are some Brazilian population groups subject \\ to endocrine disrupters?
}

Armando Meyer 1

Paula N. Sarcinelli 1

Josino C. Moreira 1

1 Centro de Estudos da Saúde do Trabalhador e Ecologia Humana, Escola Nacional de Saúde Pública Fundação Oswaldo Cruz.

Av. Brasil 4365,

Rio de Janeiro, $R J$

21045-900, Brasil.

\begin{abstract}
Several chemical substances suspected of endocrine disruption are currently being used in industrial and agricultural activities in Brazil. This paper presents analytical data showing contamination of some population groups by these substances. Virtually nothing is known about the related health effects, highlighting the need to implement a national program to evaluate such impacts.

Key words Endocrine Disrupters; Environmental Exposure; Occupational Exposure

Resumo Várias substâncias químicas com atividade sobre o sistema endócrino têm sido amplamente utilizadas no Brasil tanto em atividades industriais quanto em outros ramos, como por exemplo, na agricultura. Alguns dados mostrando a contaminação de grupos populacionais brasileiros por substâncias supostamente possuidoras destas propriedades são apresentados neste trabalho. Entretanto, muito pouco, ou mesmo nada, se sabe sobre os efeitos que as mesmas têm sobre a saúde destas populações expostas, mostrando claramente a necessidade urgente de implementação de um programa destinado a esta finalidade.
\end{abstract}

Palavras-chave Disruptores Endócrinos; Exposição Ambiental; Exposiçnao Ocupacional 


\section{Introdução}

A atividade estrogênica de substâncias químicas presentes no ambiente, denominadas estrogênios ambientais, vem sendo descrita na literatura há mais de 25 anos (Bitman \& Cecil, 1970; Nelson et al., 1978; McLachlan, 1980, 1985; Hertz, 1985; Richardson \& Bowron, 1985). Mesmo assim, só recentemente este tema tem despertado maior atenção, uma vez que somente agora estudos científicos têm comprovado a seriedade e a diversidade dos problemas que podem advir da exposição a estas substâncias. Estudos atuais têm associado esta exposição a problemas de saúde tais como aumento de certos tipos de cânceres de mama e/ou do trato reprodutivo, redução da fertilidade masculina, anormalidades no desenvolvimento sexual entre outros. Fatos, como por exemplo a deterioração da qualidade do sêmen humano (redução no número de espermatozóides, no volume médio, etc.), observados nos últimos 50 anos (Carlsen et al., 1992) e a rapidez com que estas alterações têm sido verificadas fortalecem a hipótese que atribui a origem destes problemas à exposição ambiental em detrimento daquela que atribui aos mesmos, causas genéticas (Carlsen et al., 1993).

A extensa bibliografia recentemente publicada e os vários congressos e reuniões científicas que têm sido realizados nos últimos três anos sobre este tema demonstram a atualidade bem como a importância do mesmo (Colborn \& Clement, 1992; Colborn et al., 1993, 1996; Davis et al., 1993; Davis \& Bradlow, 1995; Sharpe \& Skakkebaek, 1993; Wolff et al., 1993; Birnbaum, 1994; Kelce et al., 1994; Makela et al., 1994; Purdom et al., 1994; Rolland et al., 1995; McLachlan \& Korach, 1995; UBA, 1995; Kavlock et al., 1996; EPA, 1997; JETOC, 1997; Carmichel, 1998; IPCS, 1998; IPCS/OECD, 1998).

\section{Substâncias químicas com ação disruptora sobre o sistema endócrino}

Embora algumas substâncias naturais de origem vegetal possuam propriedades endócrinas - os fitoestrógenos (encontrados em algumas sementes, frutos ou vegetais) -, estas geralmente não causam tantos problemas ao homem quanto aquelas de origem antropogênica porque não se ligam fortemente aos receptores hormonais, são facilmente excretadas, não se acumulando nos tecidos corpóreos. As substâncias sintéticas com ação disruptora, por outro lado, são geralmente persistentes no ambiente, acumulam-se no solo e nos sedimentos, são transportadas facilmente para outras regiões pela atmosfera e podem se acumular ao longo da cadeia trófica, expondo os animais superiores a maiores riscos. Várias destas substâncias são excretadas por meio do leite materno, constituindo-se, assim, uma fonte de contaminação de recém-nascidos.

Entretanto, o conhecimento sobre o papel destas substâncias no aparecimento de doenças, sobre as relações dose-resposta, bem como sobre as metodologias utilizadas nestes estudos constituem ainda um desafio mesmo para países industrializados (EPA, 1997).

Uma relação das substâncias químicas suspeitas ou comprovadamente possuidoras de ação disruptora sobre o sistema endócrino é mostrada na Tabela 1.

Como pode ser observado, diversas substâncias bastante conhecidas e utilizadas no Brasil nos mais variados ramos de atividades, algumas já amplamente disseminadas no ambiente como resultado de atividades antropogênicas, têm sido referidas como possuidoras de atividade disruptora sobre o sistema endócrino.

Algumas como as bifenilas policloradas (PCBs), os ftalatos, as dibenzodioxinas/furanos policlorados, os alquilfenóis, o bisfenol A e vários pesticidas merecem atenção. Os PCBs, por exemplo, foram até há alguns anos, largamente utilizados em todo o mundo como isolantes e anti-inflamáveis em equipamentos elétricos tais como transformadores, em adesivos e plastificantes, sendo posteriormente proibidos em função de seus efeitos sobre a saúde ambiental e humana. Mesmo assim, ainda hoje vários problemas têm sido causados por estas substâncias químicas devido à grande persistência das mesmas no ambiente, por deficiência nos procedimentos de desativação dos estoques e por problemas de estocagem e destruição. Estes compostos estão amplamente distribuídos no mundo e a exposição aos mesmos se dá principalmente pela ingestão de alimentos.

As dibenzodioxinas e os dibenzofuranos policlorados, ainda que não sejam produtos principais, são obtidos em vários processos industriais de síntese de compostos orgânicos clorados, por exemplo, a produção de PVC, de alguns pesticidas como o ácido 2,4,5-triclorofenoxiacético -2,45-T, bem como na produção de papel ou em processos de incineração não controlados de material orgânico ou misturas contendo cloro.

Uma estimativa da concentração de dioxinas possivelmente presentes no solo da Cidade dos Meninos, Município de Duque de Caxias, RJ, feita pelo Laboratório de Toxicologia do Centro de Estudos da Saúde do Trabalhador e 
Substâncias químicas com larga distribuição no ambiente e com relatos científicos de ação sobre o sistema endócrino - disruptores endócrinos - (adaptado de Colborn et al., 1993).

\begin{tabular}{ll}
\hline Tipo & Substâncias \\
\hline Pesticidas & Herbicidas: 2,4-D; 2,4,5-T; Alacloro; Amitrole; Atrazina; Metribuzin; \\
& Nitrofen; Trifluralin. \\
& Fungicidas: Benomil; Hexaclorobenzeno; Mancozeb; Maneb; \\
& Metiram; Zineb; Ziram. \\
& Inseticidas: $\beta$-HCH; Carbaril; Clordano; Dicofol; Dieldrin; DDT \\
& e metabólitos; Endosulfan; Heptacloro; Lindano ( $\gamma$-HCH); \\
& Metoxicloro; Mirex; Oxiclordano; Paration; Piretróides; Toxafeno. \\
& Nematocidas: Aldicarb; DBCP. \\
& Cádmio, Dioxina (2,3,7,8-TCDD), Chumbo, Mercúrio, PBB's, PCB's, \\
nonilfenóis, Ftalatos, Estirenos.
\end{tabular}

Ecologia Humana (CESTEH) com base no teor de dioxinas normalmente encontrado em resíduos de $\mathrm{HCH}$, revelou um valor de $800 \mathrm{ng}$ ITeq/kg de solo. Nesta região, moram cerca de 1000 pessoas, algumas há mais de 40 anos, em contato direto e contínuo com cerca de 300 toneladas de $\mathrm{HCH}$ abandonados após desativação de uma fábrica governamental que produzia este composto. Até recentemente (1994) nesta área situava-se ainda um Abrigo para crianças abandonadas com cerca de 800 crianças. Concentrações bastante elevadas de alguns isômeros de $\mathrm{HCH}$ (principalmente do isômero beta) foram detectadas em todos os segmentos humanos e ambientais nesta região (Oliveira et al., 1995; Braga, 1996). É interessante ressaltar que no Estado de Baden Württemberg, Alemanha, por exemplo, solos com teores superiores a $100 \mathrm{ng} / \mathrm{kg}$ devem ser totalmente trocados em casos de contato com crianças (Ministerium fur Unwelt, 1992).

O Bisfenol A é um ingrediente comumente encontrado nos “vernizes" empregados em tratamentos dentários e em revestimentos internos de recipientes para embalagem de alimentos, o que facilita a contaminação humana diretamente ou por ingestão dos alimentos contaminados. Possui ação estrogênica comprovada sobre cultura de células de câncer de mama (Gascon et al., 1997).

A ação estrogênica de vários pesticidas vem sendo motivo de preocupação. Alguns organoclorados como o DDT e seus metabólitos DDD e DDE, o metoxicloro, o dieldrin e o mirex são reconhecidos como comprovadamente possuidores desta ação. Entretanto a lista de pesticidas suspeitos de interagirem com o sistema en- dócrino é bem mais extensa, como podemos observar na Tabela 1. Para se ter uma idéia, de uma relação de 107 substâncias citadas na literatura como possíveis disruptores endócrinos 64 (60\%) são pesticidas (JETOC, 1997).

\section{Os pesticidas com ação disruptora}

Sabe-se que o uso de pesticidas tem aumentado continuamente desde 1940 e hoje em dia estima-se um consumo anual superior a $3 \mathrm{mi}$ lhões de toneladas desses agentes, o que envolve um montante de mais de 20 bilhões de dólares (Pimentel, 1991). Os países em desenvolvimento e os de economia em transição respondem pelo consumo de cerca de $25 \%$ da produção mundial de pesticidas.

Dados do Sindicato Nacional da Indústria de Defensivos Agrícolas (SINDAG) mostram que o mercado brasileiro vem crescendo significativamente desde 1991 (Figura 1), quando movimentou cerca de US\$ 988 milhões. Em 1997 esta cifra subiu para cerca US\$ 2,2 bilhões, o que representa um incremento de cerca de $130 \%$.

Um aumento de cerca de $17 \%$ foi observado nos primeiros 5 meses de 1998 quando comparados com igual período de 1997.

Se considerarmos apenas os pesticidas sob suspeitas de possuírem ação disruptora do sistema endócrino, verifica-se que a utilização dos mesmos tem crescido consideravelmente nos últimos cinco anos. Apenas de portos americanos, em 1996, foram exportados 33.136 toneladas destes compostos, ou seja, cerca de 90 toneladas por dia. Os principais destinos des- 
Figura 1

Estimativa de venda de pesticidas, em dólares, no mercado brasileiro - 1991/97.

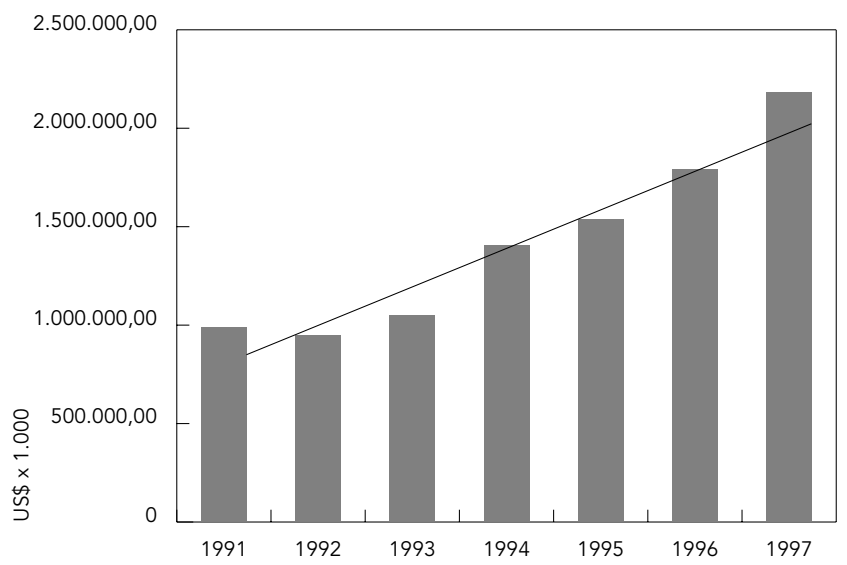

tas exportações foram Bélgica (8.664 ton $26 \%$ ), Brasil (2.645 ton - 8\%), Argentina (1.778,5 ton $-5,3 \%)$, Índia $(1.550,5$ ton $-4,6 \%)$. É importante lembrar que a Bélgica não é provavelmente o destino final destas substâncias e o Brasil, como segundo maior destino, aparece com destaque no consumo destes agentes (FASE, 1998).

Uma das substâncias mais estudadas sob o aspecto de disrupção endócrina é o DDT (e seus metabólitos DDD e DDE). Embora ainda existam muitas controvérsias e discrepâncias entre os resultados obtidos em vários estudos, este composto é sabidamente persistente no meio ambiente já tendo seu uso, produção e venda proibidos em cerca de 80 países. No Brasil, a partir de 1982, sua utilização foi legalmente restrita a campanhas de saúde pública quando era utilizado no combate ao Aedes, vetor da malária. Este procedimento foi utilizado até recentemente (1994), quando se optou por uma mudança na estratégia do combate à propagação desta enfermidade. Dados da Fundação Nacional de Saúde mostram que a região Amazônica concentra cerca de 98\% dos casos de malária no Brasil e que durante o período de 1988 a 1994, cerca de 3.000 toneladas de DDT foram utilizadas em campanhas de combate ao vetor desta doença. Estes fatos podem explicar os elevados níveis de DDT encontrados no soro de 89 índios da tribo dos Parka-Tejê, que vivem cerca de $50 \mathrm{~km}$ a leste de Marabá, Estado do Pará. Concentrações médias de 52 ppb (faixa de concentração de 12,8 a 262,6 ppb e des- vio padrão de 46,79$)$ foram encontradas nestas amostras $(0,17$ ppb para população não exposta) (Koifman et al., 1998).

A grande estabilidade destes compostos à degradação ambiental associada às suas propriedades físico-químicas (lipofilicidade, etc.) favorecem suas distribuições pelos diferentes compartimentos ambientais e suas propriedades de bioacumulação em tecidos ricos em lipídios, constituindo-se assim uma importante via de contaminação humana. De fato, a avaliação da concentração plasmática de pesticidas organoclorados realizada em 22 gestantes ainda no primeiro trimestre de gravidez, atendidas no Hospital Gafrée Guinle, Rio de Janeiro, RJ, mostrou contaminação por DDT e seus metabólitos DDE e DDD em 90\% dos casos (faixa de concentração de 0,27 a $0,44 \mathrm{ppb}$ ), por hexaclorociclohexanos - isômeros $\alpha, \beta$ e $\gamma-\mathrm{HCH}-$ em $88 \%$ (faixa de concentração: $\alpha$ de 0,17 a 1,41 ppb, $\beta$ de 0,08 a 0,81 ppb e $\gamma$ de 0,07 a 0,28 ppb), por hexaclorobenzeno em $77 \%$ (faixa de concentração: de 0,008 a 0,58 ppb), por aldrin em $41 \%$ (faixa de concentração de 0,1 a 0,17 ppb), por dieldrin em 9\% (faixa de concentração de 0,06 a $0,1 \mathrm{ppb}$ ), por endosulfan em $14 \%$ (faixa de concentração de 0,05 a 0,15 ppb) e por dodecacloro,1 caso (concentração de 2,3 ppb). Como pode ser depreendido destes resultados, a maioria das gestantes apresentava contaminação múltipla (Koifman et al., 1998). Embora estas concentrações sejam baixas, nada se conhece sobre os níveis teciduais destes compostos, que são bioacumulativos, ou sobre os efeitos que estas substâncias, isoladamente ou em conjunto, terão sobre os filhos, que ainda não podem ser avaliados.

No Brasil, a região sudeste é a que apresenta a mais alta relação entre venda de pesticidas/pessoa de todo o país, apresentando, em 1985, uma relação de cerca de 12 kg/pessoa ocupada (Garcia, 1997). Dentro da região sudeste o Estado de São Paulo se destaca com uma relação de cerca de $32 \mathrm{~kg} /$ pessoa ocupada, a maior relação de todo o país (Garcia \& Almeida, 1991).

Dados preliminares obtidos em um estudo piloto realizado nos Municípios de Magé e Nova Friburgo, RJ, mostraram que uma considerável parcela da população estudada utiliza pesticidas sob forte suspeita de ação disruptora do sistema endócrino, conforme pode ser observado na Tabela 2 .

Os efeitos que estes agentes químicos podem ter sobre a população exposta não foram ainda avaliados. 
Relação de alguns dos pesticidas de maior utilização, no Estado do Rio de Janeiro, segundo estudo piloto realizado na região em 1998.

\begin{tabular}{lll}
\hline \% de usuários & Pesticida (princípio ativo) & Aplicação (classe) \\
\hline 90 & tamaron (metamidofós)b & Inseticida/(organofosforado) \\
70 & sumidan (esfenvelerato) & Inseticida \\
60 & ditane (mancozeb)a & Fungicida (ditiocarbamato) \\
60 & gramoxone (paraquat) & Herbicida (bipiridílico) \\
35 & decis (deltametrina)a & Inseticida (piretróide) \\
\hline
\end{tabular}

a pesticidas disruptores do sistema hormonal (Colborn, 1995)

b embora o metamidofós não esteja listado entre os compostos suspeitos de tal efeito, existe uma formulação de

tamaron (tamaron`EP), que utiliza também paration, este sim suspeito de causar disrupção do sistema endócrino.

\section{Conclusão}

Associando-se a precariedade da forma com que, de maneira geral, as substâncias químicas são utilizadas em nosso País com a utilização simultânea de várias delas, geralmente em grandes quantidades, verifica-se a existência de risco elevado que pode se tornar num espaço de tempo curto, até mesmo de uma geração, um problema de gravíssimas conseqüências para a Saúde Pública e até mesmo para o desenvolvimento nacional.

Dados disponíveis, como aqueles aqui apresentados, mostram a contaminação de grupos populacionais brasileiros por substâncias químicas persistentes sobre as quais existe a suspeita de apresentarem propriedades disruptoras. Tais dados são, quase que exclusivamente, relacionados a compostos organoclorados, a maioria dos quais utilizados na agricultura. Estes compostos, desde a muito, têm suas utiliza-

\section{Referências}

BIRNBAUM, L. S., 1994. Endocrine effects of prenatal exposure to PCBs, dioxins, and other xenobiotics: Implications for policy and future research. Environmental Health Perspectives, 102:676-679.

BITMAN, J. \& CECIL, H. C., 1970. Estrogenic activity of DDT analogs and polychlorinated biphenyls. Journal of Agriculture and Food Chemistry, 18: 1108-1112.

BRAGA, A. A. M. C. B., 1996. Avaliação da Contaminação Humana por HCH em Residentes e Estudantes da Cidade dos Meninos, Duque de Caxias, RJ. Dissertação de Mestrado, Rio de Janeiro: Escola Nacional de Saúde Pública, Fundação Oswaldo Cruz.

CARLSEN, E.; GIWERCMAN, A.; KEIDING, N. \& SKAKKEBAEK, N. E., 1992. Evidence for decreasing quality of semen during past 50 years. British Medical Journal, 305:609-613. ções restritas e regulamentadas por leis específicas. Contudo, devido à sua elevada persistência no ambiente, estas substâncias são bastante encontradas no organismo humano. Atualmente, o uso destes compostos tem diminuído consideravelmente tanto devido às restrições legais quanto ao desenvolvimento de novas substâncias químicas mais eficientes e mais facilmente degradadas no ambiente. Infelizmente também paira sobre várias destas substâncias a suspeita de apresentarem propriedades endócrinas.

No Brasil, a ignorância sobre o manejo adequado das substâncias químicas e as condições de vida do homem do campo fazem com que os trabalhadores rurais possam ser apontados como um grupo prioritário para a implementação urgente de um programa com o objetivo de avaliar o impacto destas substâncias sobre a saúde do homem e do ambiente nas diversas regiões brasileiras, particularmente naquelas com elevada atividade agrícola.

CARLSEN, E.; GIWERCMAN, A.; KEIDING, N. \& SKAKKEBAEK, N. E., 1993. Evidence for increasing incidence of abnormalities of the human testis: A review. Environmental Health Perspectives, 101(Sup. 2):65-71.

CARMICHEL, H., 1998. Sex offenders. Chemistry in Britain, 34:25-29.

COLBORN, T. \& CLEMENT, C., 1992. Chemically Induced Alterations in Sexual and Functional Development: The Wildlife/Human Connection. Princeton: Princeton Scientific Publishing Co, Inc.

COLBORN, T.; VOM SAAL, F. S. \& SOTO, A. M., 1993. 1993. Developmental effects of endocrine-disrupting chemicals in wildlife and humans. Environmental Health Perspectives, 101:378-384.

COLBORN, T.; DUMANOSKI, D. \& MYERS, J. P., 1996. Our Stolen Future: Are We Threatening Our Fertili- 
ty, Intelligence, and Survival? A Scientific Detective Story. New York: Dutton Books.

DAVIS, D. L. \& BRADLOW, H. L., 1995. Can environmental estrogens cause breast cancer? Scientific American, 273:166-172.

DAVIS, D. L.; BRADLOW, H. L.; WOLFF, M.; WOODRUFF, T.; HOEL, D. G. \& ANTON-CULVER, H., 1993. Medical hypothesis: Xenoestrogens as preventable causes of breast cancer. Environmental Health Perspectives, 101:372-377.

EPA (U.S. Environmental Protection Agency), 1997. Special Report on Environmental Endocrine Disruption: An Effects Assessment and Analysis. EPA/ 630/R-96/012. Risk Assessment Forum. Washington, D.C.: EPA.

FASE (Foundation for Advancements in Science and Education), 1998. Exporting Risks - Pesticide Exports from US Ports, 1995-1996. Los Angeles: FASE.

GARCIA, E. G., 1997. Pesticide control experiences in Brazil. Pesticide Safety, 2:5.

GARCIA, E. G. \& ALMEIDA, W. F., 1991. Exposição dos trabalhadores rurais aos agrotóxicos no Brasil. Revista Brasileira de Saúde Ocupacional, 72:7-11.

GASCON, J.; OUBINA, A. \& BARCELO, D., 1997. Detection of endocrine disrupting pesticides by enzyme-linked immunosorbent assay (ELISA): Application to atrazine. Trends in Analytical Chemistry, 16:554-562.

HERTZ, R., 1985. The estrogen problem. Retrospect and prospect. In: Estrogens in the Environment II. Influences on Development (J. A. McLachlan, ed.), pp. 1-11, New York: Elsevier.

IPCS (International Program for Chemical Safety), 1998. Report of Second IPCS Steering Group Meeting on Endocrine Disruptors. Ispra: IPCS.

IPCS/OECD (International Program for Chemical Safety/Organization for Economic Co-Operation and Development), 1998. Report of IPCS/OECD Scoping Meeting on Endocrine Disruptors. Washington, D.C.: IPCS/OECD.

JETOC (Japan Chemical Industry Ecology-Toxicology and Information Center), 1997. A Study on Hormone-Like (Hormone-Mimic) Effects of Exogenous Substances. Japan Chemical Industry Association. June, 1997. Tokyo.

KAVLOCK, R. J.; DASTON, G. P.; DEROSA, C.; FENNER-CRISP, P.; GRAY, L. E.; KAATTARI, S.; LUCIER, G.; LUSTER, M.; MAC, M. J.; MACZKA, C.; MILLER, R.; MOORE, J. ROLLAND, R.; SCOTT, G.; SHEEHAN, M.; SINKS, T. \& TILSON, H. A., 1996. Research needs for the risk assessment of health and environmental effects of endocrine disruptors: A report of the U.S. EPA-sponsored workshop. Environmental Health Perspectives, 104 (Sup. 4):715-740.

KELCE, W. R.; MONOSSON, E.; GAMCSIK, M. P.; LAWS, S. C. \& GRAY Jr., L. E., 1994. Environmental hormone disruptors: Evidence that vinclozolin developmental toxicity is mediated by antiandrogenic metabolites. Toxicology and Applied Pharmacology, 126:276-285.
KOIFMAN, S.; FERRAZ. I.; VIANA, T. S.; SILVEIRA, C. L. P.; CARNEIRO, M. T. D.; KOIFMAN, R. S.; SARCINELLI, P. N.; MATTOS, R. C. O. C.; LIMA, J. S.; SILVA, J. J. O.; MOREIRA, J. C.; FERREIRA, M. F. A.; FERNANDES, C. \& BULCÃO, A. C., 1998. Cancer cluster among young indians living near power transmission lines in Bom Jesus do Tocantins, Pará, Brazil. Cadernos de Saúde Pública, 14 (Sup. 3):161-172.

MAKELA, S.; DAVIS, V. L.; TALLY, W. C.; KORKMAN, J.; SALO, L.; VIHKO, R.; SANTTI, R. \& KORACH, K. S., 1994. Dietary estrogens act through estrogen receptor-mediated processes and show no antiestrogenicity in cultured breast cancer cells. Environmental Health Perspectives, 102:572-578.

McLACHLAN, J. A., 1980. Estrogens in the Environment. Amsterdã: Elsevier Press.

McLACHLAN, J. A., 1985. Estrogens in the Environment II. Influence on Development. Amsterdã: Elsevier Press.

McLACHLAN, J. A. \& KORACH, K. S., 1995. Symposium on estrogens in the environment, III. Environmental Health Perspectives, 103 (Sup. 7):3-4.

MINISTERIUM FUR UMWELT BADE-WURTTEMBERG, 1992. Erlaß An Die Regierungsprasidien, Az: 44-8810.30/80.

NELSON, J. A.; STRUCK, R. F. \& JAMES, R., 1978. Estrogenic activities of chlorinated hydrocarbons. Journal of Toxicology and Environmental Health, 4:325-339.

OLIVEIRA, R. M.; BRILHANTE, O. M.; MOREIRA, J. C. \& MIRANDA, A. C., 1995. Contaminação por $\mathrm{HCH}$ em área urbana da região Sudeste do Brasil. Revista de Saúde Pública, 29:228-233.

PIMENTEL, D., 1991. Diversification of biological control strategies in agriculture. Crop Protection, 10: 243-253.

PURDOM, C. E.; HARDIMAN, P. A.; BYE, V. J.; ENO, N. C.; TYLER, C. R. \& SUMPTER, J. P., 1994. Estrogenic effects of effluents from sewage treatments works. Chemistry and Ecology, 8:275-285.

RICHARDSON, M. L. \& BOWRON, J. M., 1985. The fate of pharmaceutical chemicals in the aquatic environment. Journal of Pharmacy and Pharmacology, 37:1-12.

ROLLAND, R.; GILBERTSON, M. \& COLBORN, T., 1995. Environmentally induced alterations in development: A focus on wildlife. Environmental Health Perspectives, 103 (Sup. 4):1-106.

SHARPE, R. M. \& SKAKKEBAEK, N. E., 1993. Are oestrogens involved in falling sperm counts and disorders of the male reproductive tract? Lancet, 341:1392-1395.

UBA (UMWELTBUNDESAMT), 1995. Endocrinically Active Chemicals in the Environment. Berlin: UBA.

WOLFF, M. S.; TONIOLO, P. G.; LEE, E. W.; RIVERA, M. \& DUBIN, N., 1993. Blood levels of organochlorine residues and risk of breast cancer. Journal of the National Cancer Institute, 85:648-652. 